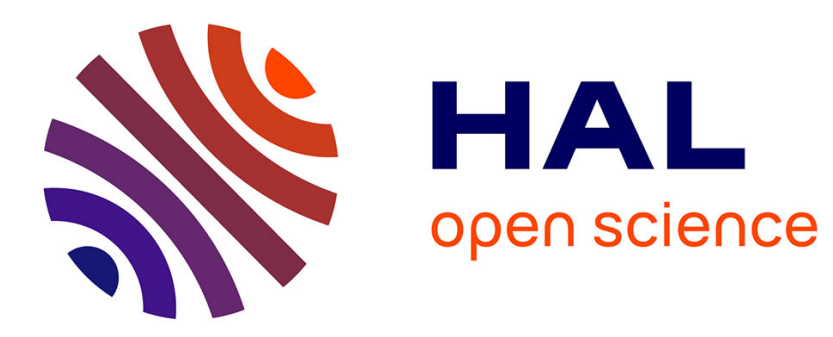

\title{
The vehicle routing problem with time windows and evidential service and travel times: A recourse model.
}

Tekwa Tedjini, Sohaib Afifi, Frédéric Pichon, Eric Lefevre

\section{To cite this version:}

Tekwa Tedjini, Sohaib Afifi, Frédéric Pichon, Eric Lefevre. The vehicle routing problem with time windows and evidential service and travel times: A recourse model.. 16th European Conference on Symbolic and Quantitative Approaches to Reasoning with Uncertainty, ECSQARU 2021, Sep 2021, Prague, Czech Republic. pp.381-395. hal-03521749

\author{
HAL Id: hal-03521749 \\ https://hal.science/hal-03521749
}

Submitted on 11 Jan 2022

HAL is a multi-disciplinary open access archive for the deposit and dissemination of scientific research documents, whether they are published or not. The documents may come from teaching and research institutions in France or abroad, or from public or private research centers.
L'archive ouverte pluridisciplinaire HAL, est destinée au dépôt et à la diffusion de documents scientifiques de niveau recherche, publiés ou non, émanant des établissements d'enseignement et de recherche français ou étrangers, des laboratoires publics ou privés. 


\title{
The vehicle routing problem with time windows and evidential service and travel times: A recourse model
}

\author{
Tekwa Tedjini ${ }^{1}$, Sohaib Afifi ${ }^{1}$, Frédéric Pichon $^{1}$, and Eric Lefèvre ${ }^{1}$ \\ ${ }^{1}$ Univ. Artois, UR 3926, Laboratoire de Genie Informatique et d'Automatique de l'Artois \\ (LGI2A), F-62400 Béthune, France \\ firstname. lastnameduniv-artois.fr
}

\begin{abstract}
This paper addresses a variant of the vehicle routing problem with time windows where service and travel times are modeled within the framework of belief function theory. This theory is general as it offers to model several facets of information imperfection, including uncertainty and imprecision. An extension of stochastic programming with recourse is used to tackle the problem. This approach aims to regain the feasibility of the routes that missed one or more of the customer time windows due to the uncertain nature of the problem. A memetic algorithm is devised to solve the problem on an adaptation of literature instances.
\end{abstract}

Keywords: Vehicle routing $\cdot$ Time windows $\cdot$ Recourse $\cdot$ Belief function $\cdot$ Memetic algorithm.

\section{Introduction}

The Vehicle Routing Problem with Time Windows (VRPTW) [9] is one of the most studied variants of vehicle routing problems (VRP). VRPTW routes are subject to time and capacity restrictions. Customers must be served within their time windows, vehicles have to return to the depot before its closure and their capacity must be respected. The main objective is to optimize the operating costs including the cost of vehicles and the overall traversed distances. The VRPTW is NP-hard. Indeed, even finding a feasible solution for the problem is itself NP-complete in the strong sense [15].

Usually, external factors like weather condition or unexpected road accidents affect one or more of the input parameters such as service and travel times, thus, the planned routes must account for the possible variations of those parameters. Accordingly, most of the research papers handled this issue using probability theory giving rise to the Stochastic VRPTW (SVRPTW) [11,18]. SVRPTW models are either tackled in a Chance-Constrained Programming (CCP) fashion [11,4,3], where the probabilities (chances) of time windows violations are below a given threshold, or in a Stochastic Programming with Recourse (SPR) one [11, 20,5]. In SPR models, routes are first planned, then, when actual service and travel times are revealed, corrections (so-called recourse actions) are performed on routes that are subject to time windows violations in order to regain their feasibility. For instance, when a vehicle arrives late at a customer's location, the service is dropped and a new visit will be rescheduled to serve him. Each correction induces a penalty cost that must be added to the routing costs. Both of the 
CCP and SPR approaches have their advantages and drawbacks, they can either be combined as in [6], or applied separately depending on the needs of the decision maker.

Set theory has also been used to handle the uncertainty in time parameters, yielding the Robust VRPTW (RVRPTW) [1, 8, 12]. This model assumes that uncertain parameters belong to a predefined set, i.e., parameters are known imprecisely, and provides solutions that are immunized against imprecision. This is commonly done by optimizing the routing costs considering extreme scenarios for service and travel times. Nevertheless, solutions tend to be overly conservative.

Recently, more general uncertainty reasoning frameworks have emerged in the VRP's literature to serve as complementary tools to the existent set/probabilistic approaches, among others the theory of belief function also known as evidence theory [16]. Beyond imprecision or (probabilistic) uncertainty, this theory offers to represent problem parameters that are affected by more subtle forms of information imperfection arising from partial lack of knowledge. In the context of VRPs, belief function theory was used, for the first time, to model the uncertainty on customers demands in the Capacitated VRP (CVRP) variant [7]. Extensions of the CCP and the SPR approaches were proposed yielding the Belief-Constrained Programming and the Belief Programming with Recourse approaches. In this paper, we follow this line of work by tackling the VRPTW with uncertain service and travel times represented within the belief function framework. A Belief-Constrained Programming version for the same problem was already proposed in [19]. Herein, a belief programming with recourse approach is devised. We assume that time windows are hard, i.e., no early or late services are allowed. The recourse policy is based on skipping service at the failures locations to regain routes feasibility. This problem is very challenging from a computational perspective, to our knowledge, even in the case of stochastic programming, few papers handled the SPR version of VRPTW with stochastic service and/or travel times and hard time windows $[20,5,6]$. Most of the works focused either on total or partial soft time windows due the difficulty of the problem. We also design a memetic algorithm [13] to solve an adaptation of literature instances.

The paper is organized as follows. Section 2 defines the VRPTW and recalls the fundamentals of belief function theory. In section 3 we describe the proposed recourse approach as well as some particular cases of the model. Section 4 is dedicated to the experimental results. Section 5 concludes the paper.

\section{Definitions and notations}

This section provides a brief background on the necessary tools required for our developments.

\subsection{Problem formulation}

Given a fleet of $K$ vehicles of the same capacity $Q$ and cost $M$ ( $M$ is a large value), the VRPTW is defined on a graph $G=(V, E)$, where $V=\{0,1, \ldots, n\}$ is the node set and $E=\{(i, j): i \neq j, i, j \in V\}$ is the arc set. The depot is denoted by 0 and $V_{c}=\{1, \ldots, n\}$ is the set of customers. Each node $i$ has a demand $q_{i} \leq Q$, a service 
time $s_{i}$ and a time interval (window) $\left[e_{i}, l_{i}\right]$ in which service can start, $q_{0}=s_{0}=0$ and $\left[e_{0}, l_{0}\right]=\left[0, l_{0}\right]$ is the time horizon for the depot. Time windows are considered to be hard, that is if a vehicle $k$ arrives at node $j$ earlier than $e_{j}$, the service is postponed until the opening of the time window, and arrivals after $l_{j}$ are forbidden. A non negative distance $d_{i j}$ and travel time $t_{i j}$ are associated with every arc $(i, j) \in E$. We assume that distances satisfy the triangle inequalities. A solution of VRPTW is composed of multiple routes, each one must respect the following constraints: 1) Each customer is only served once; 2) A vehicle $k$ leaves each visited customer and travels toward the next one on the route and each route starts and ends at the depot; 3 ) The total demand on any route does not exceed $Q$; 4) Finally, the service at each customer must start within his time window and vehicles must return to the depot before $l_{0}$. A formal description of those constraints can be found in [9]. The objective (1) minimizes the overall operating costs:

$$
\min \left[M . \sum_{k \in K} \sum_{j \in V_{c}} x_{0 j k}+\sum_{k \in K} \sum_{(i, j) \in E} d_{i j} x_{i j k}\right]
$$

where $x_{i j k}$ is the decision variable. It is equal to 1 if vehicle $k$ traverses arc $(i, j)$ and 0 otherwise. In (1), due to $M$ being large, the cost of vehicles is first minimized then the overall traversed distance is reduced. This means that a solution with fewer routes but higher distance is preferred over another one with more routes but lower distance.

\subsection{Belief function theory}

Belief function theory was first introduced by Shafer [16]. In this theory, the available knowledge about a variable $x$ defined on a finite set $\mathcal{X}$, known as the frame of discernment, is represented by a mass function $m^{\mathcal{X}}: 2^{\mathcal{X}} \mapsto[0,1]$ s.t. $\sum_{A \subseteq \mathcal{X}} m^{\mathcal{X}}(A)=1$ and $m^{\mathcal{X}}(\emptyset)=0 . m^{\mathcal{X}}(A)$ quantifies the part of our belief that $x \in A$ without providing any further information about $x \in A^{\prime} \subset A$. Each subset $A \subseteq \mathcal{X}$ such that $m^{\mathcal{X}}(A)>0$ is called focal element of $m^{\mathcal{X}}$. If all the focal elements $A \subseteq \mathcal{X}$ of $m^{\mathcal{X}}$ are singletons $(|A|=1)$ then $m^{\mathcal{X}}$ is called Bayesian and it is equivalent to a probability measure. If $m^{\mathcal{X}}$ has a unique focal element $A \subseteq \mathcal{X}$, i.e., $m^{\mathcal{X}}(A)=1, m^{\mathcal{X}}$ is said to be categorical and it corresponds to a set. In the remainder of this paper, a variable $x$ whose true value is known in the form of a mass function will be referred to as evidential variable. The notion of expected value, in probability theory, of a function $f: \mathcal{X} \mapsto \mathbb{R}$ related to a probability mass function $p^{\mathcal{X}}$ is extended, in belief function theory, to the notions of lower $\left(\underline{E}\left(f, m^{\mathcal{X}}\right)\right)$ and upper $\left(\bar{E}\left(f, m^{\mathcal{X}}\right)\right)$ expected values of $f$ related to a mass function $m^{\mathcal{X}}$ as follows [2]:

$$
\begin{aligned}
& \underline{E}\left(f, m^{\mathcal{X}}\right)=\sum_{A \subseteq \mathcal{X}} m^{\mathcal{X}}(A) \min _{x \in A} f(x), \\
& \bar{E}\left(f, m^{\mathcal{X}}\right)=\sum_{A \subseteq \mathcal{X}} m^{\mathcal{X}}(A) \max _{x \in A} f(x) .
\end{aligned}
$$

\section{A recourse model for the VRPTW with evidential times}

In this section, we present the VRPTW with evidential service and travel times under the recourse programming approach. Our model is inspired from the work in [7] and 
adapted to account for uncertainty in both service and travel times, the time windows requirements as well as a different recourse policy. We will start by formalizing the problem and by showing how to incorporate and compute the expected cost of recourse actions. Then, we will present an efficient method to compute these costs in presence of particular evidential information about service and travel times. Finally, we will discuss some special cases of the problem.

\subsection{Formalization}

Consider a solution to the VRPTW where time windows constraints are relaxed. Let $R=\{1, \ldots, i, \ldots, n, n+1=0\}$ be a route from this solution. For the sake of simplicity we suppose, without loss of generality, that the $i^{\text {th }}$ visit on $R$ corresponds to customer $i$ of the problem. To account for the time windows constraints, one must check the feasibility of $R$ at each of its visits: If the time needed for a vehicle to arrive at visit $i$ (customer or depot) meets its corresponding time window $\left[e_{i}, l_{i}\right]$ then the time window constraint is verified for $i$. In case where the vehicle arrives later than $l_{i}$, a failure occurs and $i$ can not be served, so the vehicle skips the service of $i$ and travels directly toward visit $i+1$ the immediate successor of $i$ on $R$. An exclusive visit will be rescheduled later to serve $i$. We adopt this policy since the actual values of service and travel times are only revealed once the vehicle arrives at the visit's location, thus failures can not be predicted. This is equivalent to the strategy used in [6] for the stochastic case. Formally, let $f_{i}$ be a binary variable describing the failure situation at visit $i . f_{i}$ equals 1 if the vehicle arrives later than $l_{i}$ otherwise it is equal to 0 . Arrival times are computed recursively by cumulating service and travel times up to visit $i$ with possible truncations induced by waiting times. Formally, let $a_{i}, i=1, \ldots, n+1$ be the arrival time at visit $i$. $a_{i}$ is a function of the set $\mathcal{T}_{01} \times \mathcal{S}_{1} \times \ldots \times \mathcal{S}_{i-1} \times \mathcal{T}_{i-1 i}$ of all service and travel times up to $i$, where $\mathcal{S}_{i}$ (resp. $\mathcal{T}_{i-1 i}$ ) is the set on which the service time $s_{i}$ (resp. travel time $\left.t_{i-1 i}\right)$ is defined:

$$
a_{i}= \begin{cases}v_{i-1}+t_{i-1 i} & \text { if } i \in\{2, \ldots, n+1\} \\ t_{01} & \text { if } i=1 .\end{cases}
$$

where $v_{i}$ is the departure time from visit $i$ which is expressed by:

$$
v_{i}= \begin{cases}\max \left\{e_{i}, a_{i}\right\}+s_{i} & \text { if } a_{i} \leq l_{i}, \\ a_{i} & \text { if } a_{i}>l_{i} .\end{cases}
$$

The failure situation along $R$ is represented by a unique vector $f=\left(f_{1}, \ldots, f_{n+1}\right) \in$ $\mathcal{F}=\{0,1\}^{n+1}$ in presence of a certain and precise (deterministic) knowledge about service and travel times. This vector is a function of all service and travel times on $R$ :

$$
\begin{aligned}
g: \mathcal{T}_{01} \times \mathcal{S}_{1} \times \ldots \times \mathcal{S}_{n} \times \mathcal{T}_{n n+1} & \mapsto \mathcal{F} \\
\left(t_{01}, s_{1}, \ldots, s_{n}, t_{n n+1}\right) & \mapsto f=\left(f_{1}, \ldots, f_{n+1}\right) .
\end{aligned}
$$

Each rescheduled visit induces a penalty that must be added to the routing cost of $R$. A penalty is an extra cost that measures, for instance, customers dissatisfaction or 
simply the cost of dedicating exclusive vehicles to serve them. Note that it is possible to define more complex recourse policies, nonetheless, we chose this simple policy to somehow manage the tractability of the problem given the complexity of belief functions. Let $f=\left(f_{1}, \ldots, f_{n+1}\right) \in \mathcal{F}$ be a failure vector and let $p$ be the function defined from $\mathcal{F}$ to $\mathbb{R}^{+}$, representing the penalty induced by $f$. This penalty is composed of the cost of using an extra vehicle to serve visit $i$, whose service has been skipped, plus the total distance of a round trip from $i$ to the depot:

$$
p(f)=\sum_{i=1}^{n+1} f_{i} \times\left(M+2 d_{0 i}\right) .
$$

The cost $\bar{C}(R)$ of a route $R$ in presence of a deterministic failure vector $f$ is composed of the routing cost $C(R)$ plus the penalty $p(f)$ induced by $f$ :

$$
\begin{aligned}
\bar{C}(R) & =C(R)+p(f) \\
& =M+\sum_{(i, j) \in E(R)} d_{i j}+\sum_{i=1}^{n+1} f_{i} \times\left(M+2 d_{0 i}\right) .
\end{aligned}
$$

When service and travel times are evidential, i.e., knowledge about these variables is represented by mass functions, the failure situation is no longer deterministic but evidential and it is represented by a mass function $m^{\mathcal{F}}$ as will be detailed in Section 3.2. In this case, the cost $\bar{C}(R)$ of a route $R$ consists of the routing cost part $C(R)$ to which is added the (lower or upper) expected value of $p$ related to $m^{\mathcal{F}}$ as recalled in Section 2.2. In this work, we assume a pessimistic attitude toward the possible failures that may occur on route $R$, and therefore choose to use the upper expectation (3). We denote this upper expected value by $\bar{E}\left(p, m^{\mathcal{F}}\right)$ :

$$
\bar{E}\left(p, m^{\mathcal{F}}\right)=\sum_{F \subseteq \mathcal{F}} m^{\mathcal{F}}(F) \times \max _{f \in F} p(f) .
$$

The cost $\bar{C}(R)$ of a route $R$ is then expressed as:

$$
\begin{aligned}
\bar{C}(R) & =C(R)+\bar{E}\left(p, m^{\mathcal{F}}\right) \\
& =\underset{(i, j) \in E(R)}{M+\sum_{i j}+\bar{E}\left(p, m^{\mathcal{F}}\right) .}
\end{aligned}
$$

Consequently, the overall cost $\bar{C}(S)$ of a solution $S=\left\{R_{1}, \ldots, R_{K}\right\}$ of the VRPTW with evidential service and travel times under the recourse approach, is nothing but the sum of all the expected costs of its $K$ routes.

$$
\bar{C}(S)=\sum_{k \in K} \bar{C}\left(R_{k}\right)
$$

\subsection{Evidential failures}

In this section, we start by describing how evidential knowledge about service and travel times induces evidential failures that are represented by a mass function. Then, 
we present an efficient method to compute this mass function under some particular assumptions.

Let us first consider the route $R$ defined in Section 3.1 and suppose that service $s_{i}$ and travel $t_{i j}$ times are evidential and represented, respectively, by mass functions $m_{s_{i}}^{\mathcal{S}_{i}}$ and $m_{t_{i j}}^{\mathcal{T}_{i j}}$ defined on the sets $\mathcal{S}_{i}$ and $\mathcal{T}_{i j}$, such that $\left|\mathcal{S}_{i}\right| \leq \mathcal{S} \in \mathbb{N}_{+}^{*}$ and $\left|\mathcal{T}_{i j}\right| \leq \mathcal{T} \in \mathbb{N}_{+}^{*}$. Assume that service and travel time variables are independent (this assumption is not required but only stated here to simplify the explanation of our method), hence, the joint mass function $m^{\mathcal{T}_{01} \times \mathcal{S}_{1} \times \ldots \times \mathcal{S}_{n} \times \mathcal{T}_{n n+1}}$ of service and travel times on $R$ is equal to the product of independent mass functions $m_{s_{i}}^{\mathcal{S}_{i}}$ and $m_{t_{i j}}^{\mathcal{T}_{i j}}$, for all $i \in V_{c}(R)$ and $(i, j) \in E(R)$. When $m^{\mathcal{T}_{01} \times \mathcal{S}_{1} \times \ldots \times \mathcal{S}_{n} \times \mathcal{T}_{n n+1}}$ has a unique focal set $T \subseteq \mathcal{T}_{01} \times \mathcal{S}_{1} \times$ $\ldots \times \mathcal{S}_{n} \times \mathcal{T}_{n n+1}$ such that $m^{\mathcal{T}_{01} \times \mathcal{S}_{1} \times \ldots \times \mathcal{S}_{n} \times \mathcal{T}_{n n+1}(T)=1 \text {, knowledge about the }}$ failure situation is imprecise, i.e., all we know is that it belongs to a set of possible failures denoted by $F$, which is the image of the set $T$ by function $g$ defined in (6). $F$ can be described as:

$$
F=g(T)=\bigcup_{\left(t_{01}, s_{1}, \ldots, s_{n}, t_{n n+1}\right) \in T} g\left(t_{01}, s_{1}, \ldots, s_{n}, t_{n n+1}\right) .
$$

In general, when $m^{\mathcal{T}_{01} \times \mathcal{S}_{1} \times \ldots \times \mathcal{S}_{n} \times \mathcal{T}_{n n+1}}$ has at most $c \in \mathbb{N}_{+}^{*}$ focal sets, knowledge about the failure situation is both imprecise and uncertain, and can be represented by the mass function $m^{\mathcal{F}}$ defined as:

$$
m^{\mathcal{F}}(F)=\sum_{g(T)=F} m^{\mathcal{T}_{01} \times \mathcal{S}_{1} \times \ldots \times \mathcal{S}_{n} \times \mathcal{T}_{n n+1}}(T), \forall F \in \mathcal{F} .
$$

To compute $m^{\mathcal{F}}$ defined in (13), $g(T)$ is evaluated for each focal set $T \subseteq \mathcal{T}_{01} \times$

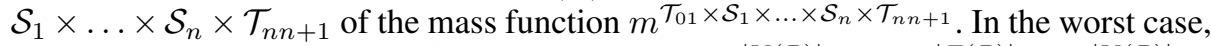
$T$ can have up to $\mathcal{S}^{n} \times \mathcal{T}^{n+1}$ element $\left(|T|=\left|\mathcal{S}_{i}\right|^{|V(R)|} \times\left|\mathcal{T}_{i j}\right|^{|E(R)|} \leq \mathcal{S}^{|V(R)|} \times\right.$ $\mathcal{T}^{|E(R)|} \leq \mathcal{S}^{n} \times \mathcal{T}^{n+1}$ ), thus evaluating (13) requires a worst case time complexity of $\mathcal{O}\left(\left[\mathcal{S}^{n} \times \mathcal{T}^{n+1}\right] \times c\right)$ which is intractable. However, following a similar tree-based approach to that of [7, Section 3.2.3] described in the next paragraph, if service and travel time variables are modeled by intervals, i.e., $s_{i} \in \llbracket \sigma_{i} \rrbracket=\left[\underline{\sigma}_{i}, \bar{\sigma}_{i}\right] \subseteq \mathcal{S}_{i}$ and $t_{i j} \in \llbracket \theta_{i j} \rrbracket=\left[\underline{\theta}_{i j}, \bar{\theta}_{i j}\right] \subseteq \mathcal{T}_{i j}$ and $T=\llbracket \theta_{01} \rrbracket \times \llbracket \sigma_{1} \rrbracket \ldots \times \llbracket \sigma_{n} \rrbracket \times \llbracket \theta_{n n+1} \rrbracket$ is the Cartesian product of interval service and travel times along $R$, one can demonstrate that only the bounds of the intervals $\llbracket \sigma_{i} \rrbracket, i \in V(R)$ and $\llbracket \theta_{i j} \rrbracket,(i, j) \in E(R)$ has to be evaluated rather than each element of $\mathcal{S}_{i}$ and $\mathcal{T}_{i j}$. Subsequently, the complexity of evaluating (13) drops to $\mathcal{O}\left(2^{2 n+1} \times c\right)$. Furthermore, this complexity can also be decreased to $\mathcal{O}\left(2^{n} \times c\right)$ if travel times $t_{i j}$ are considered as singletons rather than intervals, that is the marginalization of $m^{\mathcal{T}_{01} \times \mathcal{S}_{1} \times \ldots \times \mathcal{S}_{n} \times \mathcal{T}_{n n+1}}$ onto $\mathcal{T}_{i j}$, for any travel time variable $t_{i j}$ is a Bayesian mass function where $\llbracket \theta_{i j} \rrbracket=\left\{\tilde{\theta}_{i j}\right\}$. As a consequence $T=\left\{\tilde{\theta}_{01}\right\} \times$ $\llbracket \sigma_{1} \rrbracket \ldots \times \llbracket \sigma_{n} \rrbracket \times\left\{\tilde{\theta}_{n n+1}\right\}$. A similar result holds when service times are singletons, that is $\llbracket \sigma_{i} \rrbracket=\left\{\tilde{\sigma}_{i}\right\}$ and $T=\llbracket \theta_{01} \rrbracket \times\left\{\tilde{\sigma}_{1}\right\} \ldots \times\left\{\tilde{\sigma}_{n}\right\} \times \llbracket \theta_{n n+1} \rrbracket$, in which case the overall complexity becomes $\mathcal{O}\left(2^{n+1} \times c\right)$.

Consider, in the following, a categorical mass function $m^{\mathcal{T}_{01} \times \mathcal{S}_{1} \times \ldots \times \mathcal{S}_{n} \times \mathcal{T}_{n n+1}}$ having a unique focal set $T=\llbracket \theta_{01} \rrbracket \times \llbracket \sigma_{1} \rrbracket \ldots \times \llbracket \sigma_{n} \rrbracket \times \llbracket \theta_{n n+1} \rrbracket$, that is, $s_{i} \in \llbracket \sigma_{i} \rrbracket, \forall i \in V_{c}$ and $t_{i j} \in \llbracket \theta_{i j} \rrbracket, \forall(i, j) \in E$. Note that arrival times (resp. departure times) are imprecise in this case: $a_{i} \in \llbracket \alpha_{i} \rrbracket=\left[\underline{\alpha}_{i}, \bar{\alpha}_{i}\right]$ (resp. $v_{i} \in \llbracket v_{i} \rrbracket=\left[\underline{v}_{i}, \bar{v}_{i}\right]$ ), where bounds $\underline{\alpha}_{i}$ and 
$\bar{\alpha}_{i}$ (resp. $\underline{v}_{i}$ and $\bar{v}_{i}$ ) are obtained by applying the recursive reasoning described below. As mentioned before, in this case, the failure situation that can be encountered by a vehicle is described by a set $F$ defined by (12). In the following, we adapt the evaluation procedure in [7, Section 3.2.3] to our problem in order to efficiently compute $F$. Suppose that the vehicle operating on $R$ travels from visit $i-1$ to visit $i$. The arrival time $a_{i}$ at visit $i$ induces the following three cases:

1. If $\bar{\alpha}_{i} \leq l_{i}$ : there will be no failure at visit $i$, hence $f_{i}=0$ and $v_{i} \in\left[\max \left\{e_{i}, \underline{\alpha}_{i}\right\}+\right.$ $\left.\underline{\sigma}_{i}, \max \left\{e_{i}, \bar{\alpha}_{i}\right\}+\bar{\sigma}_{i}\right]$.

2. If $\underline{\alpha}_{i}>l_{i}$ : the failure situation is "precise" and service at visit $i$ is skipped i.e., $f_{i}=1$ and $v_{i} \in\left[\underline{\alpha}_{i}, \bar{\alpha}_{i}\right]$.

3. if $\underline{\alpha}_{i} \leq l_{i}<\bar{\alpha}_{i}$ : the failure situation at visit $i$ is "imprecise", so we account for both possibilities:

(a) When the true arrival time belongs to $\left[\underline{\alpha}_{i}, l_{i}\right]$, there will be no failure at visit $i$, i.e., $f_{i}=0$ and $v_{i} \in\left[\max \left\{e_{i}, \underline{\alpha}_{i}\right\}+\underline{\sigma}_{i}, l_{i}+\bar{\sigma}_{i}\right]$.

(b) When the true arrival time belongs to $\left.] l_{i}, \bar{\alpha}_{i}\right]$, service at visit $i$ is skipped, i.e., $f_{i}=1$ and $\left.\left.v_{i} \in\right] l_{i}, \bar{\alpha}_{i}\right]$.

This reasoning is applied for all the visits of $R$, starting from the first customer up to the depot, to get all the failure situations related to the focal set $T$. This latter procedure is sketched in Algorithm 1 and its execution induces a tree of $n+1$ levels. Each level $i$ represents the potential failure situations that a vehicle encounters when reaching visit $i$. Each node of a level $i$ contains information about the failure component $f_{i}$ as well as the arrival and departure time intervals. The evaluation is extended from a level to another one, and stops after evaluating level $n+1$ which corresponds to the depot. To guarantee the feasibility of single customer routes, we assume that there is no particular reason for a vehicle to arrive late at the first visit of any route $R$, thus $f_{1}=0$. Consider a branch from the tree induced by Algorithm 1 . The concatenation of the binary failure variables $f_{i}$ from the root up to level $n+1$ induces a failure vector $f=\left(f_{1}, \ldots, f_{n+1}\right)$ describing one possible failure situation on $R$. The set of all the vectors $f$ obtained from all the branches of the recourse tree, denoted by $F$, expresses all the failures that can occur on $R$. Using a similar proof to that of [7, Proposition 6], we can show that the set $F$ verifies $F=g\left(\llbracket \theta_{01} \rrbracket \times \llbracket \sigma_{1} \rrbracket \times \ldots \times \llbracket \sigma_{n} \rrbracket \times \llbracket \theta_{n n+1} \rrbracket\right)$. Note that in case $m^{\mathcal{T}_{01} \times \mathcal{S}_{1} \times \ldots \times \mathcal{S}_{n} \times \mathcal{T}_{n n+1}}$ has multiple focal sets, then given (13) the previous reasoning is applied for each one of them as demonstrated in the following example:

Example 1. Let us illustrate Algorithm 1 by considering a route $R=\{1,2,3,0\}$, where time windows are $\left[e_{0}, l_{0}\right]=[0,200],\left[e_{1}, l_{1}\right]=[5,30],\left[e_{2}, l_{2}\right]=[40,95],\left[e_{3}, l_{3}\right]=$ $[80,120]$. The available information about service and travel times is represented by the joint mass function: $m^{\mathcal{T}_{01} \times \mathcal{S}_{1} \times \mathcal{T}_{12} \times \mathcal{S}_{2} \times \mathcal{T}_{23} \times \mathcal{S}_{3} \times \mathcal{T}_{30}}$ denoted by $m$ for simplification:

$$
\begin{aligned}
& m([15,20] \times[20,25] \times[40,50] \times[30,35] \times[10,20] \times[15,20] \times[10,15])=0.6 \\
& m([15,20] \times[20,25] \times[25,30] \times[20,30] \times[10,15] \times[15,20] \times[10,15])=0.4
\end{aligned}
$$

$\mathcal{F}=\{(0,0,0,0),(0,1,0,0),(0,0,1,0),(0,0,0,1),(0,1,1,0),(0,1,0,1),(0,0,1,1)$, $(0,1,1,1)\}$ is the set of all possible failure situations on $R$, such that $f_{1}=0$.

The first focal set induces the tree illustrated in Fig. 1 and the second one induces the tree depicted in Fig. 2. Take for instance the tree in Fig. 1, it has two branches 


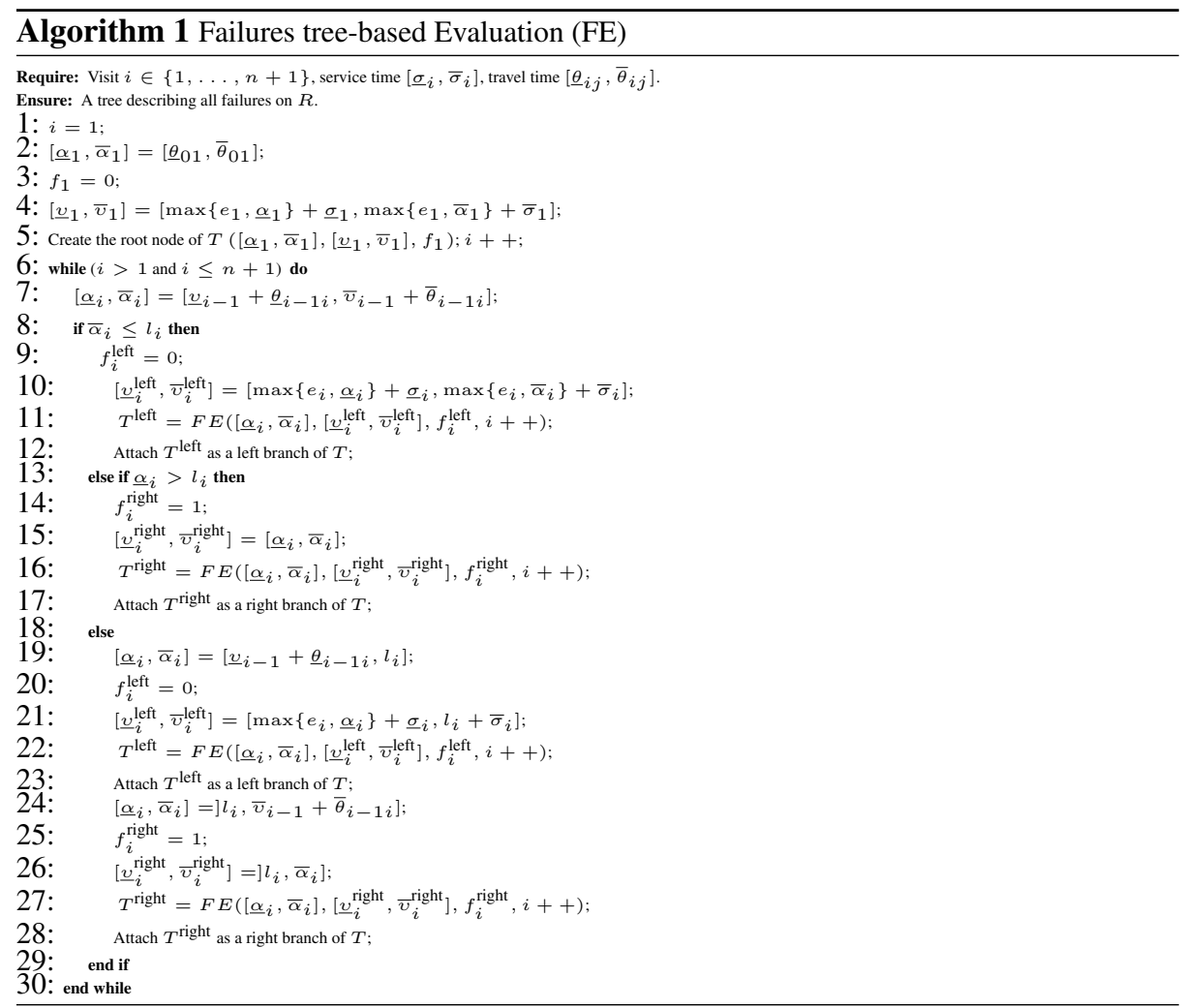

representing two possible failure situations on $R$ when information about service and travel times is given by the first focal set of $m$. The concatenation of the binary variables $f_{i}$ from the root to the leaves starting from the left branch yields the subset $F_{1}=\left\{\left(f_{1}, f_{2}, f_{3}^{1}, f_{0}^{1}\right),\left(f_{1}, f_{2}, f_{3}^{2}, f_{0}^{2}\right)\right\}=\{(0,0,0,0),(0,0,1,0)\}$, which means that either all the customers of $R$ will be successfully served or that a failure will occur at customer 3 and the latter can not be served. Similarly, the tree in Fig. 2 yields the subset $F_{2}=\left\{\left(f_{1}, f_{2}, f_{3}, f_{0}\right)\right\}=\{(0,0,0,0)\}$ with respect to the second focal set of $m$, which means that no failure will occur on $R$. Formally, using equation (13) with $g$ being the function given in (12) and $m^{\mathcal{T}_{01} \times \mathcal{S}_{1} \times \ldots \times \mathcal{S}_{n} \times \mathcal{T}_{n n+1}}$ defined such as in (14), our knowledge about the entire failure situation on $R$ can be expressed via the mass function $m^{\mathcal{F}}$ defined by:

$$
\begin{aligned}
& m^{\mathcal{F}}\left(F_{1}\right)=m^{\mathcal{F}}(\{(0,0,0,0),(0,0,1,0)\})=0.6, \\
& m^{\mathcal{F}}\left(F_{2}\right)=m^{\mathcal{F}}(\{(0,0,0,0)\})=0.4 .
\end{aligned}
$$




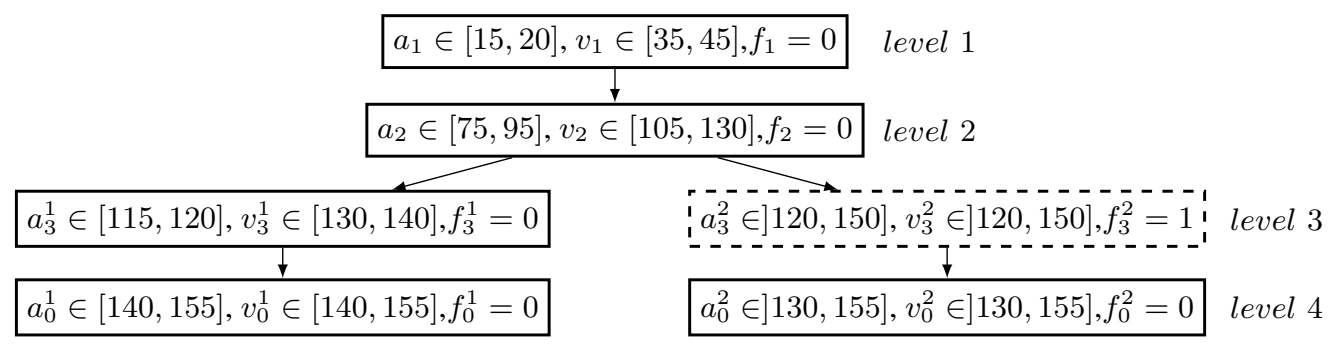

Fig. 1: The failure tree related to the first focal set of $m$.

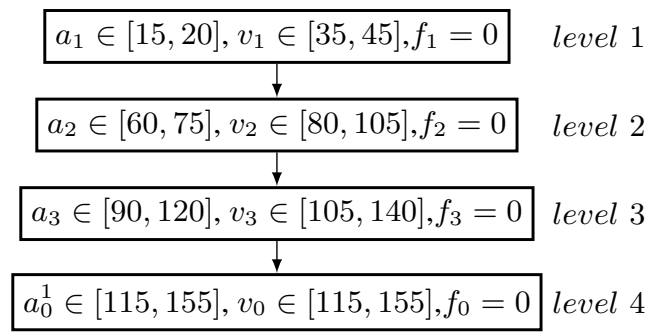

Fig. 2: The failure tree related to the second focal set of $m$.

\subsection{Particular cases}

The recourse model degenerates into well known problems when the mass function $m^{\mathcal{S}^{n} \times \mathcal{T}^{|E|}}$ representing knowledge about all service and travel time variables of the

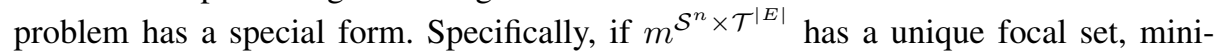
mizing the upper expected cost function (10) is equivalent to minimizing the recourse cost under the worst case scenario. Thus, our model reduces to a robust approach. Note that it is also possible to replace the upper expected penalty (9) by the lower expected one using equation (2) in case the decision maker is more interested in optimistic solutions. Another interesting particular case of the proposed model is when $m^{\mathcal{S}^{n} \times \mathcal{T}^{|E|}}$ is Bayesian, i.e., its focal sets are no longer sets but singletons. In this case, the lower and the upper expected costs of the optimal solution reduce to the classical expectation in probability theory. As a consequence, our model is equivalent to a SVRPTW with recourses. It is important to mention that the two aforementioned models (i.e., the robust and stochastic ones) are conceptually different and it is not possible, at least trivially, to convert one into another. The following example illustrates this remark.

Example 2. Consider a VRPTW instance where time windows are: $\left[e_{0}, l_{0}\right]=[0,250]$, $\left[e_{1}, l_{1}\right]=[5,20],\left[e_{2}, l_{2}\right]=[40,95],\left[e_{3}, l_{3}\right]=[80,130]$ and $Q=\infty$, vehicles unit cost is $M=1000$ and distances are given by: $d_{01}=d_{10}=10, d_{02}=d_{20}=10$, $d_{03}=d_{30}=15, d_{12}=d_{21}=20, d_{13}=d_{31}=15$ and $d_{23}=d_{32}=20$. Service and travel times are imprecise and represented by the following categorical mass function:

$$
m^{\mathcal{T}}(\{\{60\} \times\{20\} \times\{20\} \times\{10\} \times\{20\} \times\{15\} \times\{25\} \times\{30\} \times\{15,20\}\})=1
$$


with $\mathcal{T}=\mathcal{S}_{1} \times \mathcal{S}_{2} \times \mathcal{S}_{3} \times \mathcal{T}_{01} \times \mathcal{T}_{02} \times \mathcal{T}_{03} \times \mathcal{T}_{12} \times \mathcal{T}_{13} \times \mathcal{T}_{23}$.

We denote by $S_{m}^{\star}$ the optimal solution to this instance and by $\bar{C}_{m}^{*}$ its cost. The set of feasible solutions related to this instance is given in Table 1 . The solution $S_{5}$ is composed of a unique route serving customers 1,2 and 3 with a possible failure at customer 3 (highlighted in bold). From Table 1, we can deduce that the optimal solution (highlighted with *) is $S_{m}^{\star}=S_{3}$ with a corresponding cost $\bar{C}_{m}^{\star}=2060$.

Consider now another representation where service and travel times are random and expressed by a probability distribution that is compatible with $m^{\mathcal{T}}$. Denote by $S_{p}^{\star}$ its related optimal solution and by $C_{p}^{\star}$ its cost.

$$
p^{\mathcal{T}}(60,20,20,10,20,15,25,30,15)=p^{\mathcal{T}}(60,20,20,10,20,15,25,30,20)=1 / 2 .
$$

The set of feasible solutions related to $p^{\mathcal{T}}$ is given in Table 2. The optimal solution is $S_{p}^{\star}=S_{5}$ with a cost of $\bar{C}_{p}^{\star}=1580$. We clearly have $S_{m}^{\star} \neq S_{p}^{\star}$ and $\bar{C}_{m}^{\star} \neq \bar{C}_{p}^{\star}$.

\begin{tabular}{|c|c|c|c|c|}
\hline Solution & $R_{1}$ & $R_{2}$ & $R_{3}$ & Cost \\
\hline$S_{1}$ & $\{0,1,0\}$ & $\{0,2,0\}$ & $\{0,3,0\}$ & 3070 \\
$S_{2}$ & $\{0,1,2,0\}$ & $\{0,3,0\}$ & - & 2070 \\
$S_{3}^{\star}$ & $\{0,1,3,0\}$ & $\{0,2,0\}$ & - & 2060 \\
$S_{4}$ & $\{0,2,3,0\}$ & $\{0,1,0\}$ & - & 2065 \\
$S_{5}$ & $\{0,1,2, \mathbf{3}, 0\}$ & - & - & 2095 \\
\hline
\end{tabular}

Table 1: The set of feasible solutions related to the mass function $m^{\mathcal{T}}$

\begin{tabular}{|c|c|c|c|c|}
\hline Solution & $R_{1}$ & $R_{2}$ & $R_{3}$ & Cost \\
\hline$S_{1}$ & $\{0,1,0\}$ & $\{0,2,0\}$ & $\{0,3,0\}$ & 3070 \\
\hline$S_{2}$ & $\{0,1,2,0\}$ & $\{0,3,0\}$ & - & 2070 \\
\hline$S_{3}$ & $\{0,1,3,0\}$ & $\{0,2,0\}$ & - & 2060 \\
\hline$S_{4}$ & $\{0,2,3,0\}$ & $\{0,1,0\}$ & - & 2065 \\
\hline$S_{5}^{\star}$ & $\{0,1,2, \mathbf{3}, 0\}$ & - & - & 1580 \\
\hline
\end{tabular}

Note that this remark also holds with respect to our evidential model. Indeed, one can build similar examples showing that it is not possible, at least trivially, to convert the evidential service and travel times into imprecise or probabilistic ones while preserving the same solutions.

\section{Experimental results}

To solve the VRPTW with evidential service and travel times under the recourse approach, we propose to use a Memetic Algorithm (MA) [13]. This choice is motivated by MA's attractive results for VRPs and their variants $[14,10,6]$. Specifically, the MA operates on an initial set (population) of individuals or solutions that evolves by undergoing a series of modifications using some recombination and mutation tools, to create a new population, similarly as in the natural evolution process of individuals. The new population is then enhanced by exploring the neighborhoods of its individuals using the so-called local search (LS) procedures. This hybridization between population evolution and local search helps to escape local optima and provide good-quality solutions. Formally, the MA runs with a population $P O P$ of $m$ solutions. $P O P$ is first initialized and sorted increasingly according to the cost of its solutions. At each iteration, two selected parents $P_{1}$ and $P_{2}$ are crossed. The resulting offspring child has a probability $p_{m}$ to be mutated and a probability $p_{l s}$ to be enhanced by a LS procedure. child will join $P O P$ only if he improves it. The process is repeated until a stopping condition is met, such as the number of iterations without improvement of $P O P$. Note that $P O P$ 's size must be constant over the iterations. We refer the reader to $[14,10,6]$ for further 
information about selection, crossover and mutation tools. The pseudo-code of the MA is presented in Algorithm 2.

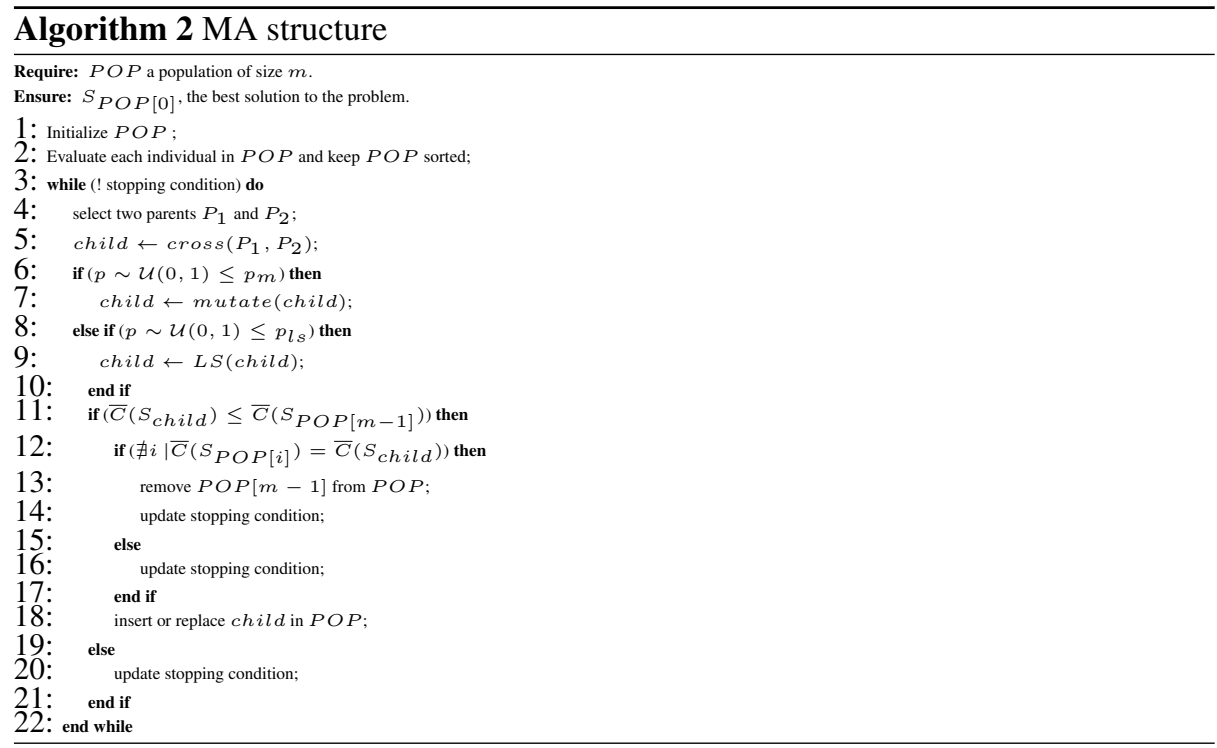

We have adapted Solomon's instances [17] to account for the evidential nature of service and travel times. These instances are composed of three sets: 25, 50 and 100 indicating the number of customers. Each set has 56 instances divided into six categories $C 1, R 1, R C 1, C 2, R 2$ and $R C 2$. Customers' positions in categories $R 1$ and $R 2$ are randomly generated meanwhile in $C 1$ and $C 2$, positions are clustered. Categories $R C 1$ and $R C 2$ gather both random and clustered positions. Another classification can be established according to the time windows nature. Categories $C 1, R 1$ and $R C 1$ (type 1) have tight time windows while categories $C 2, R 2$ and $R C 2$ (type 2) have larger time windows. We kept the same data as in the original instances, except for service and travel times which are adapted to the belief function framework as follows:

$$
m^{\mathcal{S}_{i}}\left(\left[s_{i}^{\text {det }}, s_{i}^{\text {det }}\right]\right)=0.8, m^{\mathcal{S}_{i}}\left(\left[s_{i}^{\text {det }}, s_{i}^{\text {det }}+\sigma_{i}\right]\right)=0.2
$$

where $s_{i}^{\text {det }}$ is the (deterministic) value of $s_{i}$ in Solomon's instances and $\sigma_{i}$ a random parameter generated from the interval $[1,10]$, and:

$$
m^{\mathcal{T}_{i j}}\left(\left[t_{i j}^{\text {det }}, t_{i j}^{\text {det }}\right]\right)=0.8, m^{\mathcal{T}_{i j}}\left(\left[t_{i j}^{\text {det }}, t_{i j}^{\text {det }}+\theta_{i j}\right]\right)=0.2
$$

with $t_{i j}^{d e t}$ the deterministic value of $t_{i j}$ and $\theta_{i j}$ a random parameter generated from the interval $[1,20]$. The vehicle cost $M$ is set to 1000 as in [6]. This large value prioritizes the optimization of the number of vehicles followed by the total distance and the penalties. The MA performs 15 tests for each instance of the problem and stops after $n^{2}$ iterations without improvement, with $n$ being the number of customers per instance. 
The population size is set to $n$. The LS procedure runs for $n$ iterations with a probability of 0.2 whereas, the probability of mutation is fixed to 0.01 . Explicit details about the initialization algorithm, the LS procedure as well as the parameters setting are not provided here because of the space limit. Table 3 displays average results per category for both instances of 50 and 100 customers. We recorded, for each category, the average number of vehicles $\# \bar{V}$, the average traversed distance $\overline{D i s t}$, the average total penalties $\overline{P e n}$ as well as the average execution time $(C P U(s))$ in seconds. The conducted ex-

Table 3: Average results per category

\begin{tabular}{|c|c|c|c|c|c|c|c|}
\hline \multirow[t]{2}{*}{ Category } & \multicolumn{3}{|c|}{50 customers } & \multicolumn{4}{|c|}{100 customers } \\
\hline & $\overline{D i s t}$ & $\overline{P e n}$ & $C P U(s)$ & $\# \bar{V}$ & $\overline{\text { Dist }}$ & $\overline{P e n}$ & $C P U(s)$ \\
\hline$\overline{C 1}$ & $\begin{array}{ll}6.16 & 490.16\end{array}$ & 9.49 & 6.11 & 12.04 & 1128.81 & 5.06 & 134.10 \\
\hline$C 2$ & $2.78 \quad 436.78$ & 1.79 & 16.99 & 4.67 & 759.42 & 0.00 & 326.26 \\
\hline$R 1$ & 11.881164 .98 & 751.71 & 15.13 & 22.312 & 2054.62 & 1578.82 & 252.28 \\
\hline$R 2$ & $3.24 \quad 784.21$ & 66.96 & 78.88 & 5.80 & 1246.85 & 165.32 & 2000.22 \\
\hline$R C 1$ & 10.411080 .51 & 601.26 & 13.21 & 21.55 & 2216.88 & 1063.47 & 233.30 \\
\hline$R C 2$ & $\begin{array}{ll}3.70 & 770.51\end{array}$ & 91.90 & 112.40 & 6.58 & 1449.87 & 188.59 & 1431.39 \\
\hline
\end{tabular}

periments show that the overall costs are quite acceptable given the execution time. We notice that instances of type 1 have higher costs, either in terms of $\# \bar{V}, \overline{D i s t}$ or $\overline{P e n}$, than those of type 2 (we clearly see that in classes $R 1$ and $R C 1$ ). This increase is natural and justified by the fact that these particular instances have tighter time windows, hence solutions tend to require many vehicles and they are also subject to many failures. Another remark can be established regarding the execution time. We note that CPU time is quite pronounced in type 2 instances, specifically, when the number of customers is large, this is related to the fact that this class has larger time windows, consequently, the search space is larger and requires further exploration than its counterpart of type 1.

\section{Conclusions}

We proposed a recourse approach for the VRPTW with evidential service and travel times. The model is inspired from [7] and adapted to take account of the uncertainty on both service and travel times as well as the additional hard requirements on time windows. A skipping-based policy was used to recover the feasibility of routes. Our model reduces to robust and stochastic cases when the mass functions of service and travel times are, respectively, categorical and Bayesian and allows, beyond sole imprecision or probabilistic uncertainty, to model more complex information that are affected by other forms of imperfections. We devised a memetic algorithm to solve an adaptation of literature instances. Combining both the Belief-Constrained Programming and the recourse approaches in one model as in SVRPTW [6] is an interesting and challenging perspective. 


\section{References}

1. Agra, A., Christiansen, M., Figueiredo, R., Hvattum, K.L., Poss, M., Requejo, C.: The robust vehicle routing problem with time windows. Comput Oper Res 40, 856-866 (2013)

2. Denoeux, T.: Analysis of evidence-theoretic decision rules for pattern classification. Pattern Recognition 30(7), 1095-1107 (1997)

3. Ehmke, J.F., Campbell, A.M., Urban, T.L.: Ensuring service levels in routing problems with time windows and stochastic travel times. Eur. J. Oper. Res 240(2), 539-550 (2015)

4. Errico, F., Desaulniers, G., Gendreau, M., Rei, W., Rousseau, L.M.: The vehicle routing problem with hard time windows and stochastic service times. EURO Journal on Transportation and Logistics 7(3), 223-251 (2018)

5. Errico, F., Desaulniers, G., Gendreau, M., Rei, W., Rousseau, L.: A priori optimization with recourse for the vehicle routing problem with hard time windows and stochastic service times. Eur. J. Oper. Res 249(1), 55-66 (2016)

6. Gutierrez, A., Dieulle, L., Labadie, N., Velasco, N.: A multi-population algorithm to solve the vrp with stochastic service and travel times. Computers and Industrial Engineering 125, 144-156 (2018)

7. Helal, N., Pichon, F., Porumbel, D., Mercier, D., Lefevre, E.: The capacitated vehicle routing problem with evidential demands. Int J Approx Reason 95, 124-151 (2018)

8. Hu, C., Lu, J., Liu, X., Zhang, G.: Robust vehicle routing problem with hard time windows under demand and travel time uncertainty. Comput Oper Res 94, 139-153 (2018)

9. Kallehauge, B.: Formulations and exact algorithms for the vehicle routing problem with time windows. Comput Oper Res 35(7), 2307-2330 (2008)

10. Labadi, N., Prins, C., Reghioui, M.: A memetic algorithm for the vehicle routing problem with time windows. Rairo operations Research 42, 415-431 (2008)

11. Li, X., Tian, P., Leung, S.C.: Vehicle routing problems with time windows and stochastic travel and service times: Models and algorithm. International Journal of Production Economics 125(1), 137-145 (2010)

12. Lu, D., Gzara, F.: The robust vehicle routing problem with time windows: Solution by branch and price and cut. Eur. J. Oper. Res 275(3), 925-938 (2019)

13. Moscato, P., Cotta, C.: A gentle introduction to memetic algorithms. In: Glover, F., Kochenberger, G. (eds.) Handbook of Metaheuristics, ISOR, vol. 57, pp. 105-144. Springer, Boston, MA (2003)

14. Prins, C.: A simple and effective evolutionary algorithm for the vehicle routing problem. Comput Oper Res 31(12), 1985-2002 (2004)

15. Savelsbergh, M.: Local search in routing problems with time windows. Annals of Operations Research 4(1), 295-305 (1985)

16. Shafer, G.: A mathematical theory of evidence. Princeton University Press (1976)

17. Solomon, M.: Algorithms for the vehicle routing and scheduling problem with time window constraints. Operations Research 35(2), 254-265 (1987)

18. Taş, D., Dellaert, N., van Woensel, T., de Kok, T.: Vehicle routing problem with stochastic travel times including soft time windows and service costs. Comput Oper Res 40(1), 214-224 (2013)

19. Tedjini, T., Afifi, S., Pichon, F., Lefevre, E.: A belief-constrained programming model for the VRPTW with evidential service and travel times. In: Proc. of 28 es rencontres francophones sur la Logique Floue et ses Applications. pp. 217-224. Alès, France (2019)

20. Wang, X., Regan, A.C.: Assignment models for local truckload trucking problems with stochastic service times and time window constraints. Transportation Research Record 1771(1), 61-68 (2001) 